\title{
SIX-YEAR EXPERIENCE OF CARING FOR FORTY-FOUR PATIENTS WITH A LEFT VENTRICULAR ASSIST DEVICE AT HOME: SAFE, ECONOMICAL, NECESSARY
}

David L. S. Morales, MD

Katharine A. Catanese, MSN

David N. Helman, MD

Mathew R. Williams, MD

Alan Weinberg, MS

Daniel J. Goldstein, MD

Eric A. Rose, MD

Mehmet C. Oz, MD
Objective: With increasing numbers of implantations, left ventricular assist device programs can put a financial strain on a hospital unless an efficient and safe outpatient program is developed. However, the left ventricular assist device is not widely recognized in the medical community as being reliable enough to support a patient at home. We reviewed our experience with these patients at home to assess the safety and the benefits of such a program. Methods: Our institutional 6-year experience with 90 consecutive recipients of a wearable left ventricular assist device was analyzed. Results: Forty-four $(49 \%)$ of the 90 patients who received TCI vented-electric left ventricular assist devices (Thermo Cardiosystems, Inc, Woburn, Mass) were discharged, spending a total of 4546 days (12.5 years) at home with an average of $103 \pm 16$ days of outpatient support (range 9-436 days). Of these 44 patients, all were successfully bridged to transplantation (42 patients, $96 \%$ ) or planned explantation ( 2 patients, $4 \%$ ). None of the outpatients died. The cumulative events per outpatient month were $\mathbf{0 . 0 2 0}$ for bleeding, $\mathbf{0 . 0 5 3}$ for device infection, $\mathbf{0 . 0 0 6 8}$ for thromboembolus, and 0.020 for major malfunctions. Our estimated average cost to bridge a patient to transplantation or explantation once discharged is $\$ \mathbf{1 3 , 2 0 0}$ and as an inpatient over the same length of time, including only room and board, is $\$ 165,200$. Thirty percent of outpatients were able to return to work or school, $33 \%$ to sexual activity, and $44 \%$ to driving. All outpatients performed activities of daily living. Conclusion: Current left ventricular assist device technology provides effective and economical outpatient support and is associated with limited morbidity and a satisfactory quality of life. (J Thorac Cardiovasc Surg 2000;119:251-9)
$T$ he economic value of a 103-day hospitalization, our average bridge to transplantation time, for a patient with a left ventricular assist device (LVAD) who is selfsufficient and wants to leave the hospital is unclear. With increasing numbers of implantations, LVAD programs can put a financial strain on a hospital and a

From the Department of Surgery, Columbia University, College of Physicians and Surgeons, New York, NY.

Read at the Twenty-fifth Annual Meeting of The Western Thoracic Surgical Association, Olympic Valley (Lake Tahoe), Calif, June 23-26, 1999.

Received for publication June 29, 1999; revisions requested Sept 7, 1999; revisions received Oct 19, 1999; accepted for publication Oct 21, 1999

Address for reprints: David L. S. Morales, MD, Department of Cardiothoracic Surgery, MHB 7-435, 177 Fort Washington, New York, NY 10032 (E-mail: dlm36@ Columbia.edu).

Copyright $(9) 2000$ by Mosby, Inc.

$0022-5223 / 2000 \$ 12.00+0 \quad \mathbf{1 2 / 6 / 1 0 4 0 4 2}$ health care system unless an efficient and safe outpatient program is developed.

Fortunately, prolonged hospital discharge for patients with an LVAD has become possible for several reasons: First, confidence in the devices has allowed patients, their families, and health care providers to be more comfortable with outpatient therapy. Second, the devices have become durable enough to allow extended durations of support. Third, the perception of the LVAD has changed from a last-resort therapeutic option to that of a rehabilitation-enhancing, patient-empowering device. ${ }^{1}$ As LVAD technology has matured, programs are now being asked to evaluate patients' outcomes such as satisfaction, functional capabilities, and quality-oflife issues, as well as to assess the raw survival statistics of their programs. ${ }^{2-7}$

The literature on LVAD outpatients is sparse, with the largest reported experience of LVAD patients discharged home consisting of 21 patients accumulated 
Table I. Criteria for discharging LVAD patients*

1. Patient must be hospitalized for at least 30 days after LVAD implantation

2. Patient must currently be in New York Heart Association functional class I

3. There is echocardiographic evidence indicating that the patient's native heart has sufficient contractility to open the aortic valve and maintain an arterial pressure with the LVAD operating at its lowest rate

4. Patient must have passed the required training course in the care and operation of the device

5. Patient must be accompanied by a trained companion who has passed the required training course in the care and operation of the device

6. Patient must have in the immediate vicinity required primary and backup equipment at all times

*These criteria were used for all the patients in this study; however, with the FDA approval of the HeartMate VE LVAD, we have implemented new criteria.

from the 4 most experienced LVAD programs. ${ }^{8}$ The LVAD is not widely recognized in the medical community as a device reliable enough to support a patient at home. We reviewed our 6-year experience with LVAD outpatients to assess the safety and the cost-effectiveness of such a program.

\section{Methods}

The medical records of all patients receiving a TCI (Thermo Cardiosystems, Inc, Woburn, Mass) vented-electric (VE) LVAD between February 1993 and January 1999 were reviewed. Forty-four patients were eligible for the study because they were discharged home with a TCI VE LVAD in place. Our discharge criteria have been described in previous articles (Table I). ${ }^{1,9,10}$ Discharging a patient to a rehabilitation center, to medically supervised housing, on a day trip, or home overnight did not qualify patients for this study. Inclusion of only patients discharged home without restriction allowed us to assess the safety and economics of outpatient LVAD care as though a permanent device had been implanted.

LVAD support outcomes were categorized as transplantation, explantation, or death. Outpatient morbidity was considered to be those complications that arose while the patient was discharged from the hospital. Complications that arose in the hospital but were carried over into outpatient support were not considered. A bleeding event was defined as the need to transfuse, admit, or operate on a patient because of hemorrhage associated with the LVAD. Device-related infections were defined as a positive culture from the LVAD that necessitated medical or surgical intervention. LVAD endocarditis was defined as an infection of the pseudointimal layer of an LVAD resulting in positive LVAD lining and blood cultures necessitating 6 weeks of intravenous antibiotics. A non-device-related infection was a positive culture from any non-device source accompanied by a white cell count over
$12,000 / \mathrm{mm}^{3}$ or a temperature greater than $100.5^{\circ} \mathrm{F}$ that clinically required antibiotics. Right-sided heart failure was defined as the need for inhaled nitric oxide or a right ventricular assist device. Any stroke, transient ischemic attack, neuropathy, or encephalopathy was considered a neurologic event. No systemic anticoagulation in the form of heparin or warfarin sodium (Coumadin) was given to the LVAD outpatients. Antiplatelet therapy in the form of aspirin was given to several outpatients. Any end-organ dysfunction caused by an embolus was considered a thromboembolic event. A major malfunction was defined as a pump malfunction, resulting in hemodynamic compromise and/or necessitating LVAD replacement. A minor malfunction was defined as an occurrence necessitating only a repair or replacement of the extracorporeal device, such as the controller or the battery system. Some of these complications resulted in unscheduled hospitalizations. Hospitalizations for scheduled admissions that were not a result of outpatient therapy, but for evaluations that either an outpatient or inpatient would have required, were not counted as readmissions.

Costs of all supplies were priced according to a Drug Topics Redbook written by Medical Economics Company (Montvale, NJ, 1998). ${ }^{11}$ Items not included in this book were priced by averaging their cost at 3 surgical supply stores in the New York City area. The professional fees represent the prices set by the American Medical Association in the 1999 current procedural terminology codes. ${ }^{12}$ In-hospital costs were gathered from the financial and billing departments at Columbia-Presbyterian Medical Center. All data on sexual activity, working, and driving were collected by personal interview. Results on the incidence of these three activities used the number of people engaging in these activities before LVAD implantation as the denominator.

All results are reported as a mean \pm the standard error. The 95\% confidence intervals for the incidence per outpatient were calculated by the Exact Method for Binomial Proportions. The 95\% confidence intervals for the incidence of event per outpatient month were calculated by the Exact Method for Poisson Parameters.

\section{Results}

Fifty-three (59\%) of the 90 patients who received TCI VE LVADs were released from the hospital. Nine patients were allowed day trips only and 44 (49\%) were discharged home from the hospital completely. Our results will focus on these 44 patients (91\% male) whose mean age was $46 \pm 2$ years. This group spent a total of 4546 days ( 12.5 years) at home with an average of $103 \pm 16$ days of outpatient support (median 71 days; range 9-436 days). This cohort spent $62 \% \pm 4 \%$ of their LVAD support time as outpatients. All but 4 of these 44 patients were sent home before the Food and Drug Administration (FDA) approval of the TCI VE LVAD in September of 1998.

The outcomes of the 44 patients with LVADs dis- 
Table II. Outcomes of patients with the TCI VE LVAD

\begin{tabular}{lccccc}
\hline Status & No. of patients & Transplant recipients & Death & Explantation & Ongoing \\
\hline Discharged* LVAD recipients & 44 & $42(96 \%)$ & 0 & $2(4 \%)$ & 0 \\
Nondischarged LVAD recipients & 46 & $20(44 \%)$ & $19(41 \%)$ & $2(4 \%)$ & $5(11 \%)$ \\
Total No. of VE LVADs & 90 & $62(69 \%)$ & $19(21 \%)$ & $4(4 \%)$ & $5(6 \%)$ \\
\hline
\end{tabular}

*Does not include overnight or day trips.

Table III. Complications of LVAD outpatient care

\begin{tabular}{lllccc}
\hline Type of complication & $\begin{array}{c}\text { Incidence } \\
\text { per outpatient }\end{array}$ & \multicolumn{1}{c}{$95 \%$ CI } & $\begin{array}{c}\text { Incidence of event } \\
\text { per outpatient month }\end{array}$ & $95 \%$ CI & $\begin{array}{c}\text { Resulting mortality } \\
\text { from complication }\end{array}$ \\
\hline Bleeding & $0.068(3 / 44)$ & $0.014-0.190$ & 0.020 & $0.004-0.059$ & 0 \\
Operate for bleeding & $0.045(2 / 44)$ & $0.006-0.160$ & 0.013 & $0.002-0.048$ & 0 \\
Stroke/TIA & 0 & $0-0.081$ & 0 & $0-0.025$ & N/A \\
Thromboembolic event & $0.023(1 / 44)$ & $0.001-0.120$ & 0.007 & $0-0.037$ & 0 \\
Device-related infection & $0.180(8 / 44)$ & $0.082-0.330$ & 0.053 & $0.023-0.110$ & 0 \\
Admit for device infection & $0.140(6 / 44)$ & $0.052-0.270$ & 0.040 & $0.015-0.087$ & 0 \\
Operate for device infection & 0 & $0-0.081$ & 0 & $0-0.025$ & N/A \\
Non-device-related infection & $0.091(4 / 44)$ & $0.025-0.217$ & 0.026 & $0.007-0.069$ & 0 \\
Right heart failure & 0 & $0-0.081$ & 0 & $0-0.025$ & N/A \\
Major malfunction & $0.068(3 / 44)$ & $0.014-0.190$ & 0.020 & $0.004-0.059$ & 0 \\
Minor malfunction & $0.460(20 / 44)$ & $0.300-0.610$ & 0.440 & $0.430-0.460$ & 0 \\
Admit for minor malfunction & $0.023(1 / 44)$ & $0.001-0.120$ & 0.007 & $0-0.037$ & 0 \\
Unscheduled hospitalization & $0.270(12 / 44)$ & $0.150-0.430$ & 0.120 & $0.071-0.190$ & 0 \\
\hline
\end{tabular}

CI, Confidence interval; TIA, transient ischemic attack.

charged home and the remaining 46 patients with LVADs not discharged home are listed in Table II. No LVAD outpatients died. All outpatients were successfully bridged to transplantation or planned explantation.

The average wait for a heart in the outpatients receiving transplants was $148 \pm 21$ days. The average postoperative length of stay after transplantation for the outpatients who had LVADs was $18 \pm 2$ days. Two patients had the LVAD explanted, one on LVAD support day 389 and the other on LVAD support day 101. The first patient had another LVAD placed for recurrent heart failure on postexplantation day 178. However, she underwent successful transplantation 89 days later, spending 75 of these 89 days (84\%) at home. The second patient is doing well 120 days after explantation.

Complications. A rapid response mechanism created in case of an outpatient catastrophic event was rarely implemented, because the frequency of these events or any outpatient complications in our experience has been minimal (Table III). This includes the 9 patients allowed day trips only, who had no complications while out of the hospital or as a result of being out of the hospital.

Bleeding events. Three of our outpatients had bleeding complications, 2 requiring surgery. In 2 patients holes developed in the outflow grafts on the 46th and 352 nd days of LVAD support. Both patients were brought to the clinic, were noted to have decreased hematocrit values, and were subsequently taken to the operating room on a nonemergency basis for outflow graft replacement or repair. The third patient had a selflimited episode of bleeding on the 64th day of LVAD support from the driveline tract after sexual activity. This patient was admitted for 3 days of observation and was discharged without intervention.

Neurologic/thromboembolic events. Despite no systemic anticoagulation, no strokes or transient ischemic attacks occurred in this cohort of patients. However, 1 patient did have a microembolization to the retina, causing a transient scotoma of the left eye 83 days after implantation. This patient was admitted for 3 days of observation during which there was no intervention or anticoagulation since no source of emboli was found. This deficiency resolved almost completely and did not inhibit the patient from such activities as snowboarding.

Device-related infections. Eight patients had a device-related infection while at home. Three of the 5 patients with driveline infections were admitted to the hospital because of fevers and increased white cell 
counts while receiving oral antibiotics. After successful intravenous antibiotic therapy, 2 of these patients resumed their outpatient LVAD care and 1 patient underwent transplantation on the 7th day in the hospital. The other 2 patients with a driveline infection were treated with oral antibiotics as outpatients with no further complications. One patient had a pocket infection associated with high fevers and drainage from the abdominal wound. He was admitted and successfully treated with a 7-day course of intravenous antibiotics. Two patients with LVAD endocarditis ${ }^{13}$ were admitted with bacteremia and fever but the fever abated with intravenous antibiotic therapy. Both LVADs were explanted on those admissions, one for functional improvement of the native heart and the other for a transplant. Positive pseudointimal lining cultures at explant and pre-explant blood cultures for Pseudomonas aeruginosa and Staphylococcus epidermidis confirmed our suspicions of LVAD endocarditis.

Non-device-related infections. The immune function of patients receiving long-term LVAD support has been theorized to become deficient by the prolonged and ongoing stimulation of the immune system by the LVAD, a large antigen. Therefore we evaluated whether these LVAD outpatients contracted a higher frequency of other types of infections (ie, pneumonia, urinary tract) not directly involving the device. ${ }^{14,15}$ Only 4 non-device-related infections were identified. Two were central line infections, both necessitating admission, in patients receiving immunoglobulin $\mathrm{G}$ for positive panel reactive antibody tests. These infections resolved with line removal and intravenous antibiotics. The third incident was a Streptococcus throat infection treated on an outpatient basis and the fourth, a urinary tract infection, also treated on an outpatient basis. There was no case of pneumonia or urosepsis.

Malfunctions. A total of 66 minor malfunctions affected 20 of 44 outpatients (46\%). These included batteries not fully charging, alarms continuously sounding, and controllers malfunctioning. The vast majority of these events were managed by the patient or in the outpatient clinic. Only one of these events necessitated admission to the hospital, and this was to observe a new controller for 48 hours.

All three of the major malfunctions in our experience were recognized in the outpatient setting early and addressed in an efficient manner that avoided any clinical ramifications. There was no mortality from major malfunctions. The LVADs malfunctioned in three ways, all resulting in deficient forward flow: (1) The cam slope had broken loose, thus inhibiting the pusher plate from fully compressing the blood chamber; (2) the LVAD's ball bearings had worn after 270 days of support, making the resistance too great for the motor to work efficiently; and (3) a static shock mistakenly delivered to the LVAD system damaged the power conductor, sending the LVAD into a basal rate at 40 cycles/min. After LVAD replacement, all patients recovered without complications, were discharged, and successfully underwent transplantation after a period of outpatient support. Thus the fear that the LVAD can stop, causing sudden death at home, was not realized.

Unscheduled hospitalizations. There were 18 unscheduled hospitalizations affecting 12 of 44 (27\%) outpatients with a median hospital stay of 7 days and a range of 3 to 25 days for a total of 171 readmission days. These days were not included in the 4546 outpatient LVAD support days. These readmissions were caused by the complications discussed earlier. There were 8 scheduled admissions, lasting 1 to 4 days. All were for immunoglobulin $G$ therapy for a positive panel reactive antibody test except for one that was for a scheduled angioplasty. All of the patients who were readmitted were discharged. The 18 unscheduled readmissions resulted in discharge (15), transplantation (2), or explantation (1). None of the unscheduled hospitalizations resulted in death. Until transplantation or explantation, LVAD outpatients spent $96 \%$ of their outpatient support time at home and healthy.

Outpatient costs. The costs associated with caring for an outpatient with an LVAD are listed in Table IV, and the analysis in Table $\mathrm{V}$ shows the estimated cost of outpatient care over 1 week and over our average outpatient support time of 103 days. In our experience, the incidence of readmission per outpatient over this average time of outpatient support is 0.41 and over a week is 0.028 . The mean cost to readmit an LVAD outpatient in our hospital is $\$ 25,653 .{ }^{16}$ Using the weekly supply cost, our typical schedule for follow-up visits, and our readmission cost, we can estimate the expense of bridging our LVAD patients to transplantation or explantation once an outpatient as $\$ 13,200$. If we exclude readmissions, the cost to take care of a healthy LVAD outpatient for 1 month, including weekly clinic visits and laboratory tests, is $\$ 750$. If the FDA requirement for weekly visits is eliminated, as it has been since September of 1998, the anticipated cost per month assuming one clinic visit a month would be $\$ 600$. One day of room and board on our nonacute surgical floor costs $\$ 1604$.

Outpatient activities. The prevalence of 4 activities in particular was noted to provide a sense whether LVAD outpatients resume their "normal lives." On discharge, many patients were driving, working, going to 
Table IV. Outpatient LVAD cost

\begin{tabular}{|c|c|c|c|}
\hline Type of cost & Items (No. needed daily) & Cost & Unit cost \\
\hline Professional fee & $\begin{array}{l}\text { Clinic visit (CPT 99211) (nurse practitioner } \\
\text { or physician) }\end{array}$ & $\$ 50.00$ & $\$ 50.00$ (weekly) \\
\hline \multirow[t]{5}{*}{ Laboratory fees } & Venipuncture (CPT 36415) & $\$ 10.00$ & $\$ 76.00$ (weekly) \\
\hline & Coagulation profile & $\$ 24.00$ & \\
\hline & Hepatic profile & $\$ 20.00$ & \\
\hline & Chem 7 profile & $\$ 8.00$ & \\
\hline & $\mathrm{ABC}$ & $\$ 14.00$ & \\
\hline \multirow[t]{4}{*}{ Dressing supplies } & $4 \times 4$ gauze $(6)$ & $\$ 0.18$ & $\$ 5.17$ (daily) \\
\hline & Sterile gloves (1) & $\$ 1.14$ & \\
\hline & Nonsterile gloves (1) & $\$ 1.40$ & \\
\hline & Operation site (1) & $\$ 1.55$ & \\
\hline \multirow[t]{5}{*}{ Medications } & Ferrous sequels (2) & $\$ 0.32$ & $\$ 3.29$ (daily) \\
\hline & Antacid (Pepcid AC) $10 \mathrm{mg}$ (1) & $\$ 0.28$ & \\
\hline & Multivitamin (1) & $\$ 0.13$ & \\
\hline & Enteric aspirin (1) & $\$ 0.20$ & \\
\hline & Captopril 25 mg (3) & $\$ 0.68$ & \\
\hline
\end{tabular}

$C P T$, Current procedural terminology; $A B C$, automated blood count.

Table V. Cost to bridge LVAD outpatient to transplantation

\begin{tabular}{|c|c|c|c|c|c|}
\hline Type of cost & Unit cost & Frequency & Weekly cost & Cost per 103 days & $\begin{array}{l}\text { Monthly cost per } \\
\text { healthy outpatient }\end{array}$ \\
\hline Professional fees & $\$ 50.00$ & Weekly & $\$ 50.00$ & $\$ 735.71$ & $\$ 200.00$ \\
\hline Laboratory fees & $\$ 76.00$ & Weekly & $\$ 76.00$ & $\$ 1,118.29$ & $\$ 304.00$ \\
\hline Dressing changes & $\$ 5.17$ & Daily & $\$ 36.19$ & $\$ 532.51$ & $\$ 151.10$ \\
\hline Medications & $\$ 3.29$ & Daily & $\$ 23.03$ & $\$ 338.87$ & $\$ 98.70$ \\
\hline Readmission & $\$ 25,653$ & $0.0040 /$ day & $\$ 718.28$ & $\$ 10,462.09$ & - \\
\hline Total & N/A & N/A & $\$ 903.50$ & $\$ 13,187.47$ & $\$ 753.80$ \\
\hline
\end{tabular}

Our average length of outpatient support (103 \pm 16 days). Healthy outpatient means no readmissions. $N / A$, Not available.

school, and resuming sexual activity (Table VI). Patients returned to being lawyers, musicians, high school students, and graduate students. However, several adults did not return to work because their insurance benefits for LVAD care would have been forfeited.

Worldwide experience. Table VII shows the total worldwide LVAD outpatient experience as of January 1, 1999, not including day trips or overnight passes, according to the three main manufacturers of LVADs. ${ }^{* *}$ A total of 261 patients have been sent home with a TCI LVAD, 205 patients with a Novacor LVAD (Novacor Division, Baxter Healthcare Corporation, Oakland, Calif), and 5 patients with a Thoratec LVAD (Thoratec Laboratories, Pleasanton, Calif).

*Jacobs D. Novacor out-of-hospital experience. Personal communication, December 1998.

'Krauskopf T. Summary of patients in release program: vented electric LVAS. Personal communications, December 1998.

Marchesani G. TLC-II portable driver outpatient experience. Personal communications, December 1998.

\section{Discussion}

The reluctance to discharge patients with an LVAD stems from the innumerable problems that patients and caregivers can imagine arising at home and their possibly fatal outcomes. This hesitancy to discharge LVAD patients was seen in the first attempts at outpatient care, in which patients were discharged from the hospital but stayed in medically supervised housing. ${ }^{4,17,18}$ This is not surprising since the literature on the safety and reliability of outpatient LVAD care is sparse. The largest single outpatient program experience was reported by Columbia-Presbyterian Hospital and consisted of only 19 patients. ${ }^{1}$ Therefore published proof of the safety and reliability of outpatient LVADs is not overwhelming, as seen by a review of the literature (Table VIII). ${ }^{1,8,17-19}$ However, data for outpatient LVADs do exist, even though not reported. To this end, the FDA, after studying all the outpatient data from the manufactures, approved the only two available portable LVADs in the United States, the TCI VE HeartMate and the 
Table VI. Ability of outpatients to return to "active living"

\begin{tabular}{lccc}
\hline Activity & $\begin{array}{c}\text { No. of patients } \\
\text { involved in activity } \\
\text { before LVAD support }\end{array}$ & $\begin{array}{c}\text { No. of } \\
\text { LVAD outpatients } \\
\text { involved in activity }\end{array}$ & $\begin{array}{c}\text { Percent able to } \\
\text { return to activity }\end{array}$ \\
\hline Work/school & 44 & 13 & $30 \%$ \\
Sexually active & 30 & 10 & $33 \%$ \\
Driving & 36 & 16 & $44 \%$ \\
\hline
\end{tabular}

Table VII. World experience of LVAD patients discharged home

\begin{tabular}{lccc}
\hline Device & $\begin{array}{c}\text { No. of patients, } \\
\text { US/non-US }\end{array}$ & $\begin{array}{c}\text { Outpatient support } \\
\text { in days }\end{array}$ & $\begin{array}{c}\text { Mortality } \\
\text { as outpatient }\end{array}$ \\
\hline Novacor & $71 / 134^{*}$ & $32,157(88.1 \mathrm{y})$ & 0 \\
TCI VE LVAD & $141 / 120$ & $28,981(79.4 \mathrm{y})$ & 2 \\
Thoratec VAD & $0 / 5$ & $100(0.3 \mathrm{y})$ & 0 \\
Total & 471 & $61,237(167.8 \mathrm{y})$ & $2(0.012 / \mathrm{pt}-\mathrm{y})$ \\
\hline
\end{tabular}

Data do not include overnight or day trips. US, United States.

*Includes patients discharged to outpatient housing.

Novacor 100 W LVAD, for outpatient use in September of 1998. A third device, the Thoratec TLC II, which can serve as a right, left, or biventricular assist device, has been available for outpatient use in Canada since November 1998 and in Europe since May 1998 (personal communication from G. Marchesani, December 1998). The worldwide LVAD outpatient experience of 471 patients with a total of 168 support-years out of the hospital and a $1.2 \%$ per patient-year mortality is impressive, but unpublished. ${ }^{* \dagger}$ This mortality is a significant improvement when compared with the 66\% 1year mortality of a transplant status I patient with class IV heart failure or with the 30\% 1-year mortality of all patients on the cardiac transplant list. ${ }^{20,21}$ There are also significant improvements in regard to freedom from hospitalization. Most LVAD recipients are United Network for Organ Sharing (UNOS) status I, receiving inotropic support, and waiting in the intensive care unit with class IV heart failure. Even if these patients improve and are sent home, an average group of 44 patients with class III or IV heart failure supported for the same amount of time as our outpatient cohort would be admitted 50 times (4 times per patient-year), ${ }^{22}$ whereas our LVAD outpatients were admitted 18 times (1.4 times per patient-year). Outpatient LVAD care is

*Jacobs D. Novacor out-of-hospital experience. Personal communication, December 1998.

'Krauskopf T. Summary of patients in release program: vented electric LVAS. Personal communications, December 1998.

Marchesani G. TLC-II portable driver outpatient experience. Personal communications, December 1998. not a therapy of the future but a safe therapy for patients with end-stage heart failure that currently is being practiced in the United States and around the world.

Our experience with LVAD outpatients has been positive and our initial concerns of complications, especially device failure, occurring with no medical supervision were relieved for three main reasons. First, complications outside the hospital were infrequent, especially when compared with our in-hospital rate, as evidenced by our in-hospital non-device and devicerelated infection rates of $60 \%$ and $27 \%$, respectively, which are markedly more than our outpatient incidences of $7 \%$ and $18 \%$, respectively. ${ }^{13,23}$ Second, if they did occur, the outpatient's LVAD education, the LVAD personnel access and communication systems, and the physiologic discharge criteria of adequate left ventricular function served as safety nets. ${ }^{9,10}$ These nets allowed early detection of all complications followed by a swift and seamless plan of therapy, avoiding frantic or emergency situations out of the hospital and resulting in no outpatient mortality. Third, no complications of clinical significance occurred as a result of being an outpatient; all complications could have occurred in the hospital. Thus, whether the patients were in or out of the hospital made no difference in regard to safety. However, being an outpatient did have several benefits in terms of cost and quality of life.

Reallocation away from the inpatient setting, the most costly component of the $\$ 35$ billion spent on heart failure treatment every year, to the outpatient setting is 
Table VIII. Available literature on LVAD outpatient programs (experiences and safety)*

\begin{tabular}{llc}
\hline Program & \multicolumn{1}{c}{$\begin{array}{c}\text { No. of }^{*} \\
\text { discharged patients }^{*}\end{array}$} \\
\hline Columbia-Presbyterian Medical Center & J Am Coll Cardiol (1997) & 19 \\
Bad Oeynhausen Heart Center & Eur J Cardiothorac Surg (1997) & 6 \\
Multicenter & ASAIO (1996) & 21 \\
Pittsburgh University & Ann Thorac Surg (1996) & $6^{\ddagger}$ \\
Texas Heart Institute & ASAIO (1994) & 1 \\
Bad Oeynhausen Heart Center & Correspondence (1998; unpublished) & $36^{\ddagger}$ \\
\hline
\end{tabular}

* Discharged home (does not include day or overnight trips).

‘Texas Heart Institute, Columbia Presbyterian Medical Center, Cleveland Clinic Foundation, St Luke’s Medical Center.

Indicates some patients discharged to hospital-run housing or rehabilitation centers.

becoming a necessity in the ever increasingly cost-conscious health care system. ${ }^{20,22}$ Outpatient mechanical support may be one option in deferring hospital costs. Although the initial cost of mechanical circulatory support is expensive, the ability to streamline patients to discharge and maintain them on an out-of-hospital basis may lessen the economic burden this type of illness places on society.

An outpatient LVAD program ameliorates two main economic burdens of inpatient LVADs: one is the cost of in-hospital care and the other is the use of hospital resources, especially personnel. The latter is important because our program, especially when just beginning, had a maximum number of LVAD inpatients that could be cared for adequately because of a finite quantity of resources and of LVAD-trained personnel. The wait for donor hearts is 148 days in our overall experience, and when LVAD patients were not routinely discharged, a difficult to manage patient load would accumulate. Discharging LVAD patients when appropriate has allowed us to focus on new and critically ill LVAD patients and has allowed patients undergoing long-term LVAD support to enter into an independent phase of rehabilitation.

The second, more concrete economic benefit is reduction of overall cost for taking care of an LVAD patient after implantation. Our estimated average cost to bridge a patient to transplantation or explantation once discharged, including the cost of readmissions, is $\$ 13,200$. The cost of caring for an LVAD inpatient over the same length of time, charging only for room and board on the nonacute floor (thus excluding medicine, medical care, and professional fees), is $\$ 165,200$. Also, to care for a healthy LVAD outpatient, which is how our cohort spent $96 \%$ of their outpatient support time, for 1 month (\$750) is less expensive than the 1-day charge for room and board on our nonacute hospital service, $\$ 1600$.
The other major benefit to an outpatient program is the improvement in the quality of life when patients are discharged home. The hospital serves as a haven for sick people to be dependent on others for their care and rehabilitation. However, once rehabilitation has reached a certain level, the confines of the hospital may inhibit the independent rehabilitation a person needs to achieve a personal perception of mental, social, and physical health. Patients with pneumatic LVADs, which are not FDA approved for outpatient care, are often supported in the hospital for extended periods of time while awaiting donor hearts and frequently feel stifled and "imprisoned" by the hospital. ${ }^{18,24,25}$ However, the patients with an electrical LVAD are able to be discharged and have taken full advantage of this opportunity. All 44 LVAD outpatients performed activities of daily living independently. They also engaged in a host of activities, which included going to restaurants, the movies, and parties with friends, as well as caring for children, ice skating, bicycle riding, playing sports, playing in a band, and volunteer work. The high prevalence of the three activities listed in Table VI and the breadth of the activities listed above compel one to believe that outpatient care allowed these patients to fully complete their social, mental, and physical rehabilitation.

The above observations are based on the perceptions and opinions of the caregivers. Recently, a thorough and formal quality-of-life study on LVAD outpatients was completed by Dew and associates. ${ }^{26}$ Using the Sickness Impact Profile subclasses for physical function and for social interaction, as well as the self-report Symptom Checklist-90 (SCL-90), LVAD outpatients were demonstrated to improve in the physical, psychologic, emotional, and social aspects of their lives when discharged home and fared significantly better than transplant candidates at home or LVAD inpatients. In fact, LVAD outpatients closely resembled the trans- 
plant recipients 7 months after transplantation in physical, psychologic, and emotional well-being. ${ }^{3,26}$ Interestingly, the transplant recipients felt more of a burden to their family than did the LVAD outpatients.

Substantial evidence now exists through patients' actions and perceptions, caregivers' observations, and some formal outcome/quality-of-life research by Dew and associates that outpatient LVAD care provides a better quality of life than inpatient care. ${ }^{1,9,10,26}$

We believe that an outpatient LVAD program is safe and economical while being socially, physically, and psychologically beneficial to the patient. Our confidence in outpatient LVAD care has led to an $84 \%$ discharge rate and has resulted in a minimal complication incidence. Outpatient LVAD support is a necessary part of an LVAD program and should be sought by most cardiac mechanical assist programs.

\section{REFERENCES}

1. DeRose JJ Jr, Umana JP, Argenziano M, et al. Implantable left ventricular assist devices provide an excellent outpatient bridge to transplantation and recovery. J Am Coll Cardiol 1997;30:17737.

2. Arabia FA, Smith RG, Jaffe C, et al. Cost analysis of the Novacor left ventricular assist system as an outpatient bridge to heart transplantation. ASAIO J 1996;42:M546-9.

3. Dew MA, Kormos RL, Roth LH, et al. Life quality in the era of bridging to cardiac transplantation: bridge patients in an outpatient setting. ASAIO J 1993;39:145-52.

4. Kormos RL, Murali S, Dew MA, et al. Chronic mechanical circulatory support: rehabilitation, low morbidity, and superior survival. Ann Thorac Surg 1994;57:51-7.

5. Mehta SM, Aufiero TX, Pae WE Jr, Miller CA, Pierce WS. Mechanical ventricular assistance: an economical and effective means of treating end-stage heart disease. Ann Thorac Surg 1995; 60:284-90.

6. Moroney DA, Powers K. Outpatient use of left ventricular assist devices: nursing, technical, and educational considerations. Am J Crit Care 1997;6:355-62.

7. Moskowitz AJ, Weinberg AD, Oz MC, Williams DL. Quality of life with an implanted left ventricular assist device. Ann Thorac Surg 1997;64:1764-9.

8. Myers TJ, Catanese KA, Vargo RL, Dressier DK. Extended cardiac support with a portable left ventricular assist system in the home. ASAIO J 1996;42:M576-9.

9. Catanese KA, Goldstein DJ, Williams DL, et al. Outpatient left ventricular assist device support: a destination rather than a bridge. Ann Thorac Surg 1996;62:646-52.

10. DeRose JJ, Argenziano M, Sun BC, Reemtsma K, Oz MC, Rose EA. Implantable left ventricular assist devices: an evolving longterm cardiac replacement therapy. Ann Surg 1997;226:461-8.

11. Medical Economics Company Inc. Drug topics redbook. Montvale [NJ]; 1999.

12. Kirschner C, Davis S, Evans D, et al. Current procedural terminology. IV. Revised edition. Chicago: American Medical Association; 1977. p. 10, 148.

13. Argenziano M, Catanese KA, Moazami N, et al. The influence of infection on survival and successful transplantation in patients with left ventricular assist devices. J Heart Lung Transplant 1997; 16:822-31.

14. Massad MG, Cook DJ, Schmitt SK, et al. Factors influencing HLA sensitization in implantable LVAD recipients. Ann Thorac Surg 1997;64:1120-5.

15. Williams MR, Moazami N, Itescu S, et al. Platelet transfusions are associated with the development of anti-MHC class I antibodies in patients with left ventricular assist support [abstract]. J Heart Lung Transplant 1997;16:105.

16. Gelijns AC, Richards AF, Williams DL, Oz MC, Oliveira J, Moskowitz AJ. Evolving costs of long-term left ventricular assist device implantation. Ann Thorac Surg 1997;64:1312-9.

17. Fey O, E1-Banayosy A, Arosuglu L, Posival H, Korfer R. Out-ofhospital experience in patients with implantable mechanical circulatory support: present and future trends. Eur J Cardiothorac Surg 1997;11(Suppl):851-3.

18. Myers TJ, Dasse KA, Macris MP, Poirier VL, Cloy MJ, Frazier $\mathrm{OH}$. Use of a left ventricular assist device in an outpatient setting. ASAIO J 1994;40:M471-5.

19. Winowich B, Nastala CJ, Pristas JM, Griffith BP, Kormos RL. Discharging patients who are undergoing mechanical circulatory support. Ann Thorac Surg 1996;61:478-9.

20. Goldstein DJ, Rose EA. Cardiac allotransplantation. In: Rose EA, Stevenson LW, editors. Management of end-stage heart disease. 1st ed. Philadelphia: Lippincott-Raven; 1998. p. 177-85.

21. Stevenson LW. When is heart failure a surgical disease? In: Rose EA, Stevenson LW, editors. Management of end-stage heart disease. 1st ed. Philadelphia: Lippincott-Raven; 1998. p.129-46.

22. Robbins MA, O'Connell JB. Economic impact of heart failure. In: Rose EA, Stevenson LW, editors. Management of end-stage heart disease. 1st ed. Philadelphia: Lippincott-Raven; 1998. p. 311.

23. Levin HR, Chen JM, Oz MC, et al. Potential of left ventricular assist devices as outpatient therapy while awaiting transplantation. Ann Thorac Surg 1994;58:1515-20.

24. Frazier OH. Chronic left ventricular support with a vented electric assist device. Ann Thorac Surg 1993;55:273-5.

25. Frazier $\mathrm{OH}$. First use of an untethered, vented-electric left ventricular assist device for long-term support. Circulation 1994;89: 2908-14.

26. Dew MA, Kormos RL, Winowich B, et al. Quality of life outcomes in left ventricular assist system inpatients and outpatients. ASAIO J 1999;45:218-25.

\section{Discussion}

Dr Bruce Reitz (Stanford, Calif). This is an extraordinary, groundbreaking experience. Dr Rose and Dr Oz deserve a lot of credit for their perseverance and the excellent care that made this possible. They were able to discharge half of these 90 patients without any deaths and bring them to transplantation. Also impressive are the activities that the patients are able to engage in during this time, although I must say that snow-boarding is a little bit scary.

Dr Morales. I agree. We do not usually advise such an activity for our patients.

Dr Reitz. These impressive results also include the fact that only 1 patient had a thromboembolic event despite the fact that no anticoagulants were given to these patients. 
Clearly this is very cost effective, as those of us who are involved in assist device and transplant programs can appreciate. I think your experience is now being duplicated, although in many fewer numbers, at a number of other centers.

I would like to ask you several questions and go into a little more of the detail that was in the manuscript. First, to understand how this outpatient group was determined, you do list approximately 10 criteria that patients have to meet to become an outpatient. For example, the aortic valve has to open during a time that the LVAD is turned down to show that the patient has some cardiac output should the device abruptly fail. Which of these criteria are the most frequent ones that prevent the patient from becoming an outpatient?

Dr Morales. Thank you for your comments. At first I think it was the 30-day restriction by the FDA. Several times, we thought we could have discharged patients sooner, but we did not because of the 30-day restriction. Also, the physiologic criterion is one of the critical safety nets for the outpatient program. In the 3 patients whose devices did fail, the ability for their native heart to give them some level of perfusion allowed them to get into the hospital, have the problem be diagnosed, and then have the device exchanged.

Dr Reitz. You had 9 patients in a "day trip" mode. Do all of the patients who become outpatients go on day trips first, or to a supervised environment, before they become completely discharged to their home?

Dr Morales. Our protocol, which switched in September 1998 when the FDA approved the TCI LVADs, was that every patient would go through five 1-day trips with their companion and then five 3-day trips. After that, they could be completely discharged. Those were the patients who were eligible for this study.

Dr Reitz. I believe you said that the 9 patients who were taking day trips underwent transplantation, and that prevented them from getting to the outpatient status. Is that right?

Dr Morales. Yes, sir.

Dr Reitz. Among the 3 patients with major problems, 2 patients had graft disruptions and bleeding that necessitated reoperation. Was the graft disruption in any particular place, and is there something that could be done to prevent that?

Dr Morales. The hole developed probably as a result of wear. One was identified at 6 weeks and the other at almost a year. The Dacron graft of the TCI LVAD sometimes kinks or bends as it is going into the ascending aorta. Dr Oz believes it can rub against the thoracic cage, which then causes the graft to wear. Actually, in both of these patients the low hematocrit levels were identified during routine visits to the outpatient clinic. Further investigation, through imaging studies, showed collections of blood surrounding the grafts.

Dr Reitz. Are you saying that these were contained ruptures, and not symptomatic?

Dr Morales. Yes, sir.
Dr Reitz. The manuscript mentions that some of these patients became sensitized, with high panel reactive antibodies, which we know is a problem with some long-term LVADs. In the group that was at home, how many patients became sensitized, and what did you do to take care of that?

Dr Morales. I do not know the exact number because I considered this a scheduled admission. They would have had this same problem whether they were an inpatient or outpatient. What would usually occur in these patients is a 3-day admission for intravenous immunoglobulin $G$ therapy to combat the positive panel reactive antibodies.

Dr Reitz. Your success with the outpatients clearly supports the rationale for the permanent trial that I believe has started at your institution and several others. What is the status of that particular trial? Have you had the same kind of results so far that you presented in this report?

Dr Morales. I do not know the data of the rematch trial. I do not believe that information has been released publically. Dr Rose and Dr Oz could better answer this question.

Dr Walter Dembitsky (San Diego, Calif). We all have gone through the stage of "how can we do it." We are passing through the stage of "how should we do it," and now we are all entering the phase of "how do we do it best." In that spirit, we are looking at the same things in San Diego and we have been very pleased with our outpatient LVAD population. Are you evaluating quality-of-life indicators in these patients?

Dr Morales. We published a study I believe 3 years ago, but that was a very small cohort. Actually, the best quality-oflife study has been done by the Pittsburgh group, which I mentioned as a reference in the article. ${ }^{26}$ In June 1999 in the ASAIO Journal they published a formal quality-of-life study using different mechanisms such as the Sickness Impact Profile and the self-report Symptom Checklist-90. They have shown that LVAD patients at home actually have the same quality of life as transplant patients 7 months after receiving their allograft and believe that their quality of life is much better than that of transplant candidates or inpatient LVAD patients.

Dr Dembitsky. Thank you. Finally, on that rematch, 42 patients have been randomized.

Dr Michael Reardon (Houston, Tex). In our LVAD program we are not sending people home, but right next door at the Texas Heart Institute Dr Frasier is. Your results are extraordinarily good. These people seem to be doing very well. What with the risks of heart transplantation and immunosuppression, do any of your patients tell you that they do not want the transplant operation?

Dr Morales. Yes. One patient, an ophthalmologist, really tried to refuse his transplant because he said he was operating better than he had for the past few years before receiving the LVAD. It was quite a fight to get the LVAD out, but there was no choice. 